\title{
A narrative review of imaging tools for imaging subgingival calculus
}

\author{
Jason J. Chang ${ }^{1}$, Casey Chen ${ }^{2}$, Joe Chang ${ }^{2}$, Sreenivas Koka ${ }^{3}$, Jesse V. Jokerst ${ }^{4,5,6}$ \\ ${ }^{1}$ Department of Biological Sciences, University of California, San Diego, CA, USA; ${ }^{2}$ Division of Periodontology, Diagnostic Sciences and Dental Hygiene, \\ Herman Ostrow School of Dentistry, University of Southern California, Los Angeles, CA, USA; ${ }^{3}$ School of Dentistry, University of Mississippi Medical \\ Center, Jackson, MS, USA; ${ }^{4}$ Material Science and Engineering Program, University of California, San Diego, CA, USA; ${ }^{5}$ Department of Bioengineering, \\ University of California, San Diego, CA, USA; ${ }^{2}$ Department of Radiology, University of California, San Diego, CA, USA \\ Contributions: (I) Conception and design: JV Jokerst, JJ Chang; (II) Administrative support: JV Jokerst, S Koka; (III) Provision of study materials or \\ patients: None; (IV) Collection and assembly of data: JJ Chang; (V) Data analysis and interpretation: JJ Chang; (VI) Manuscript writing: All authors; \\ (VII) Final approval of manuscript: All authors. \\ Correspondence to: Sreenivas Koka, DDS, MS, PhD, MBA. UMMC School of Dentistry, Jackson, MS 39216, USA. Email: skoka@umc.edu.
}

Background and Objective: The conventional method of detecting subgingival calculus involves using a periodontal probe to sense tactile differences on the dental root surface. Although efficient, this method can result in false positives and false negatives. This literature review explores alternative detection techniques that can detect subgingival calculus with improved accuracy and consistency. The accumulation of dental calculus below the gingival margin can foster periodontitis-inducing bacterial growth. Conventional methods of locating subgingival calculus are often inaccurate and highly dependent on clinician skill. This literature review evaluates techniques used to improve the accuracy of imaging and detecting subgingival calculus.

Methods: Google Scholar, PubMed and PubMed Central databases were searched for peer-reviewed original articles evaluating subgingival calculus imaging and detection techniques. A total of 46 relevant articles ranging from 1981 to 2021 were included.

Key Content and Findings: This narrative review discusses the subgingival calculus detection and imaging capabilities of periodontal endoscopy in an in vivo study and of optical coherence tomography (OCT), fluorescence spectroscopy, and differential reflectometry in in vitro settings. Each technique has unique benefits and limitations that distinguishes it from the others.

Conclusions: In vitro studies have revealed that techniques including periodontal endoscopy, OCT, fluorescence spectroscopy, or differential reflectometry allow for a more accurate diagnosis of subgingival calculus deposits in comparison to detection via periodontal probing. Despite the improved results, the common limitations of these techniques include longer operation times and expensive equipment. Further studies are needed to transition these imaging and detection methods to clinical environments.

Keywords: Dental calculus; endoscopy; optical coherence tomography (OCT); fluorescence spectroscopy; differential reflectometry

Received: 26 April 2021; Accepted: 13 October 2021; Published online: 21 October 2021.

doi: $10.21037 /$ fomm-21-57

View this article at: https://dx.doi.org/10.21037/fomm-21-57

\section{Introduction}

Dental calculus (i.e., tartar) is a mineralized plaque that develops both supragingivally and subgingivally (1). Plaque is a bacterial biofilm that accumulates on teeth and can be treated by regular brushing and flossing; however, it can only be removed clinically through a procedure known as scaling and root planing (SRP). Bacterial plaque content and saliva composition are factors in calculus formation. Anatomically, calculus formation is discovered on teeth near the major salivary glands: Stenson's duct and Wharton's duct (1). Further, 
subgingival calculus is frequently found on the lingual and interproximal surfaces of mandibular teeth $(1,2)$. Not only is calculus one of the major contributing factors to periodontal diseases by elevating the levels of both viable and non-viable bacteria, it also debilitates the host inflammatory responses $(3,4)$. Periodontally, the residual calculus is positively correlated to deeper probing depths with possible clinical attachment losses $(5,6)$. Therefore, early detection and removal of calculus are critical in maintaining patient's oral health.

Detecting subgingival calculus is important in periodontal diseases prevention (2). However, the high degree of difficulty and errors may result in an inaccurate detection of subgingival calculus. Unlike subgingival calculus, supragingival calculus can be easily identified during clinical examinations: its color ranges from creamywhite to yellow or brown, distinguishing it from the surface of the enamel (7). On the other hand, considering subgingival calculus detection, practioners usually rely on tactile sensation in addition to the dental radiographs $(1,8)$.

Traditionally, a dental radiograph presents two dimensional projections of hard tissues with identifiable tooth structures including the enamel, cementum, and cementoenamel junction (CEJ) (9). Although the advancement in dental technology such as caries diagnosis, dental implant simulation, and other diagnostic aid, a traditional dental radiograph remains limited in calculus detection except the substantial interproximal tartar buildup $(8,10)$.

A common method of clinically detecting subgingival calculus is to use a dental explorer or periodontal probe to distinguish the tactile differences between the rough surface of subgingival calculus and smooth surfaces of enamel or cementum $(2,8)$. A study compared the conventional explorer probing method to the microscopic evaluation of subgingival calculus after SRP. The results showed that while clinical detection identified $19 \%$ of the root surfaces with residual calculus, microscopic evaluation found $58 \%$ of the root surfaces contained residual calculus (2). The residual calculus after instrumentation of the root surfaces were commonly found as fragments at the CEJ, in grooves, concavities, or furcation areas $(11,12)$. The residual calculus may also be present as a smooth, thin burnished layer indistinguishable from unaffected root surface by tactile sensing.

The inability to detect residual subgingival calculus leads to several adverse outcomes. When the residual calculus is left in the periodontal pocket, gingival inflammation and pathogenic recolonization activities may occur postoperatively (13). Thus, repeated instrumentation on the root surface is commonly employed to compensate for the ineffective calculus removal. However, it may inadvertently create damage to the root structures $(14,15)$. For example, rough cementum surfaces may be mistaken for calculus after repeated root surface planing $(8,16)$.

Imaging technology provides a noninvasive alternative to detecting subgingival calculus. Therefore, there have been research efforts to create objective and accurate methods to identify subgingival calculus. Generally, imaging methods can be more objective at identifying target tissue (10). This would highly benefit less experienced clinicians who are not as proficient in removing subgingival calculus (17). More importantly, imaging techniques can offer a complete depiction of the extent of disease and the response to therapy. Below, we describe the current recent research efforts in imaging subgingival calculus. We present the following article in accordance with the Narrative Review reporting checklist (available at https://fomm.amegroups. com/article/view/10.21037/fomm-21-57/rc).

\section{Methods}

Peer-reviewed original articles (written in or translated to English) evaluating subgingival calculus imaging and detection techniques were included. The explored techniques consisted of periodontal endoscopy, optical coherence tomography (OCT), fluorescence spectroscopy, and differential reflectometry. Due to the differences in imaging and detection techniques, a wide range of measurements units and protocols were employed. Articles from Google Scholar, PubMed and PubMed Central databases were searched using the terms "subgingival calculus, detection, and imaging". A total of 46 relevant articles were included and ranged from 1981 to 2021 (Table 1).

\section{Discussion}

\section{Existing imaging techniques}

Next, we review improved techniques for detecting subgingival calculus including periodontal endoscopy (13,18-20), OCT (8,21-23), laser fluorescence (12,17,24-27), two-photon autofluorescence $(3,15)$, and differential reflectometry $(10,28)$. Periodontal endoscopes identify subgingival calculus deposits through illuminating the root surfaces and transmitting the reflected light back to the eyes of the operator $(13,18-20)$. OCT produces high-resolution cross-sections of soft and hard tissues (including subgingival calculus) that can be reconstructed into a $3 \mathrm{D}$ image $(21,22,29)$. Laser fluorescence detects subgingival calculus 
Table 1 The search strategy summary

\begin{tabular}{ll}
\hline Items & Specification \\
\hline Date of search & June 17, 2020 to August 31, 2020 \\
Databases and other sources searched & Google Scholar, PubMed, PubMed Central Databases \\
Search terms used & dental calculus, subgingival calculus, detection, imaging \\
Timeframe & June 17, 2020 to October 13, 2021 \\
Inclusion and exclusion criteria & Peer-reviewed original articles, written in or translated to English \\
Selection process & JJC independently conducted the literature search with the articles reviewed by all included authors \\
\hline
\end{tabular}
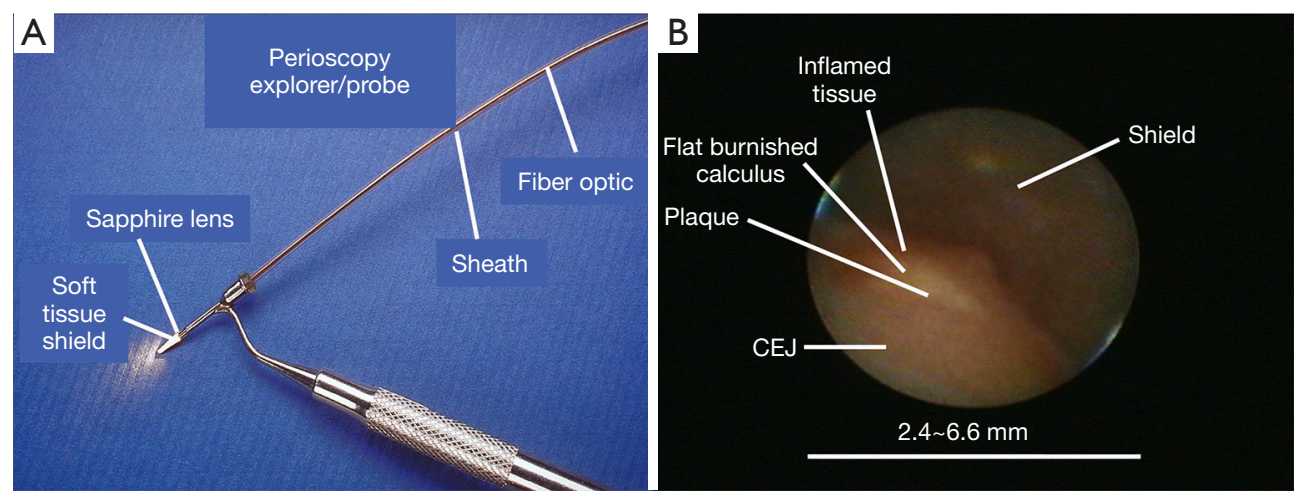

Figure 1 Perioscopy in vivo imaging of gingival sulcus. (A) A periodontal endoscope attached to a curet as modified for clinical use. The endoscopic components including the sheath, fiber optic, sapphire lens, and soft tissue shield, are listed. (B) Image produced with the dental endoscope showing visible inflamed tissue, subgingival flat burnished calculus, plaque, and CEJ. Flat burnished calculus is not tactilely detectable using a periodontal probe. Reprinted with permission from (19). Copyright 2002 John Wiley and Sons. CEJ, cementoenamel junction.

through characteristic emission wavelengths via excited root surface molecules $(17,25)$. Two-photon autofluorescence is similar to laser fluorescence except that the excitation event uses two photons rather than one for a longer excitation wavelength, better penetration depth, less scattering, and improved optical sectioning $(3,15)$. A light-emitting diode (LED)-based optical probe, DetecTar, was also designed specifically for subgingival calculus detection; it collects red LED radiation reflected from root surfaces $(10,28)$. To be clear, many of the studies focusing on these imaging and detection methods are in vitro and have yet to be clinically evaluated. However, these methods do possess potential improvement in detecting subgingival calculus compared to conventional calculus detection methods.

\section{Periodontal endoscopy}

Endoscopy is a medical technique used to visualize internal structures or crevices via fiber-optic imaging. It was first introduced in dentistry in 1999 as a diagnostic method for periodontal disease (18). Endoscopes include several hardware components, including a distal input image lens, an image transmission system, an ocular lens for image magnification and refocus, and an illumination transmission system (19). Endoscopes function via transmitting light through a set of fiber bundles to illuminate the sample and transmit reflected light out to the operator. Dental and periodontal endoscopes use a gradient lens and a fused fiber optic bundle; consequently, they are more cost-effective, more flexible, and smaller than medical endoscopes. In clinical situations, dental or periodontal endoscopes are inserted into a gingival sulcus to explore tooth landmarks, plaque, or calculus. To accommodate the oral environment, dental endoscopes, such as that in Figure 1A, also have an attached subgingival probe to provide fiber-optic imaging, a sterile sheath, an irrigation pump, an illumination lamp, a charged-coupled device (CCD) video camera for imaging the working field, and an active matrix LCD-TFT flat panel 
video display monitor for real-time viewing of the working field (not pictured) $(18,19)$. Curettes, explorers, and adaptors for ultrasonic scalers are commonly used in conjunction with dental endoscopes. The focal depth for an endoscope change from air to water; in air, the field in focus is between 1.8$5 \mathrm{~mm}$, whereas the field is between 2.4-6.6 $\mathrm{mm}$ in water (19).

Stambaugh et al. evaluated the performance of a dental endoscope in the gingival sulcus. It is important to note that the proper operation of a dental endoscope comes with a steep learning curve. Structures in endoscopic images are often difficult to identify due to the debris and fluids including blood, saliva, and water in the sulcus. The sizes of structures visualized by the endoscope are also difficult to determine. Therefore, sufficient training of 2 to 4 weeks is needed to gain the skills necessary to accurately interpret the images (19). For example, Stambaugh et al. imaged a fragment of a dark-colored subgingival calculus that appeared light in color due to the magnified light from the endoscope. Nevertheless, dental endoscopes have value in terms of observing deposits of flat, burnished calculus that could not otherwise be detected by a dental explorer or probe (demonstrated in Figure 1B) (19).

In vivo studies have been done to test the effectiveness of SRP with and without using a periodontal endoscope $(13,18)$. Geisinger et al. studied a control group undergoing a conventional SRP procedure and a test group with an SRP procedure with the assistance of a periodontal endoscope. In the control group, the patients' teeth were scaled and root planed until no calculus could be detected using a number 3 Cow Horn explorer. The patients in the test group experienced similar SRP except that a periodontal endoscope was used to detect calculus until it was fully removed (18). The teeth from both groups were then extracted, imaged using a stereomicroscope and a digital camera, and analyzed for total root surface area and percentage of total root surface area containing residual calculus using Image $\mathbf{J}(18)$. The data from the image analysis showed that the total percentage of the residual calculus found in the test group was $2.14 \% \pm 3.13 \%$ lower than that of the control group, which was statistically significant.

The periodontal endoscope was also better at deeper pocket depths. Teeth with a deeper gingival pocket in the test group had significantly less residual calculus versus the control group teeth after treatment (18). The use of periodontal endoscopes in SRP results in improved outcomes. Therefore, there is less gingival inflammation and less bleeding upon probing (13). Although more effective, the use of a periodontal endoscope requires longer treatment times versus conventional SRP. The treatment time for SRP using the periodontal endoscope for the first five patients was $26.34 \pm 5.63 \mathrm{~min}$ per tooth. However, this study also showed that treatment time decreases significantly as the operator treats more patients underscoring the learning curve associated with the use of periodontal endoscope. This treatment time using the periodontal endoscope decreased to just $15.23 \pm 3.35 \mathrm{~min}$ per tooth after treating twelve patients. In comparison, SRP treatment without the periodontal endoscope required $12.56 \pm 3.50 \mathrm{~min}$ per tooth after treating twelve patients (18).

Michaud et al. evaluated the effectiveness of a periodontal endoscope (Perioscope ${ }^{\mathrm{TM}}$, DentalView, Irvine, CA, USA) similar to Geisinger et al. with a control group undergoing conventional SRP and a test group with SRP in tandem with a periodontal endoscope. However, the difference between the two studies is that Michaud et al. included multi-rooted teeth compared to Geisinger, which only involved singlerooted teeth. Michaud et al. found that the use of periodontal endoscopes in SRP results in no significant decrease in residual subgingival calculus in multirooted molar teeth (20). For the buccal/lingual surfaces with a pocket depth less than $4 \mathrm{~mm}$, the percentages of residual calculus for the control group and test group were $14.90 \% \pm 3.94 \%$ and $14.03 \% \pm 3.46 \%$, respectively. For interproximal surfaces with pocket depths less than $6 \mathrm{~mm}$, the percentages of residual calculus for the control and test groups were $18.02 \% \pm 4.22 \%$ and $16.90 \% \pm 3.39 \%$, respectively. In both cases, of buccal/ lingual surfaces with pocket depths less than $4 \mathrm{~mm}$ and interproximal surfaces with pocket depths less than $6 \mathrm{~mm}$, there showed no statistically significant decrease in residual calculus between conventional SRP treatment and SRP treatment with a periodontal endoscope (18).

Periodontal endoscopy's potential of imaging subgingival calculus seems most promising as the technology is already available for clinical use and is also specifically designed for exploration in the gingival sulcus. The main drawback is the extended treatment time and the steep learning curve for operators.

\section{$O C T$}

OCT is a non-invasive, non-ionizing imaging method that produces high resolution, real-time cross-sections of soft and hard tissue (21). These cross-sections of tissue samples can be reconstructed or processed to produce three-dimensional images with identifiable biological microstructures $(22,29)$. OCT is predominantly used in ophthalmology and cardiology but is increasingly popular 
in dentistry (29). In dental applications, OCT can image through gingival tissue to identify tooth landmarks, caries, and subgingival calculus.

OCT imaging systems involve several key hardware components. These include a partially coherent broadband light source, an imaging apparatus, a measurement head, and a module of data processing and image generation (23). A common imaging apparatus amongst conventional OCT systems is the Michelson interferometer $(22,29)$. The interferometer splits the broadband light through a coupler, which directs the light towards the sample and reference arms. The beam is then reflected by the sample and the reference mirrors back to the coupler and then to a detector that measures the reflected signals (22). A range of backscattering and reflecting properties of the tissue sample help determine the present microstructures. OCT imaging systems generally allow for a maximum imaging depth of $2 \mathrm{~mm}$ in most biological tissues (30).

In dentistry, swept-source (SS)-OCT is typically preferred over conventional OCT. SS-OCT offers faster imaging speeds, higher sensitivity, greater detection efficiency (21), better resolution, and improved penetration depth (23). However, SS-OCT is more expensive and requires a high-speed data acquisition interface (21). Despite the many similarities between the two systems, the main key identifiers of the SS-OCT system are the swept laser source and a data acquisition interface.

The first in vivo application of OCT in dentistry was the identification of dental structures. Colston et al. designed an OCT handpiece that was more suitable for clinical environments. It was developed for intraoral examinations and could easily access the posterior portion of the oral cavity-a region highly susceptible to disease. Through using the OCT handpiece to take cross-sectional images of anterior and posterior teeth, they were able to identify the dentin, enamel, dentin-enamel junction, gingival margin, sulcus, gingival epithelium, and alveolar bone (22).

Hsieh et al. showed that in vitro SS-OCT imaging can be used to detect subgingival calculus. The SS-OCT system consisted of a fiber-based Mach-Zehnder interferometer with two couplers and two optical circulators (shown in an illustrated diagram in Figure $2 A$ and in an actual image in Figure $2 B$ ). Hsieh et al. analyzed naturally-occurring calculus on an extracted tooth with a $0.8 \mathrm{~mm}$ layer of gingival tissue (Figure 2C) and without the tissue (Figure 2D). They first used the SS-OCT system to image the uncovered sample revealing the strong presence of calculus (Figure 2E). The SS-OCT image of the covered sample shows the ability of the system to detect calculus beneath gingival tissue (Figure $2 F$ ). To image the entire tooth, 200 cross-sectional images were taken and reconstructed revealing the calculus underneath the gingival tissue. The refractive indices of the dental tissue structures were then calculated post-imaging revealing that the refractive index of the calculus of $2.112 \pm 0.127$, which is greater than that of the enamel, dentin, and cementum $(1.619 \pm 0.034$, $1.528 \pm 0.026$, and $1.567 \pm 0.030$, respectively). This comparatively high refractive index of calculus is responsible for the strong scattering property of the calculus that allowed it to be detected even below a layer of gingival tissue (21).

In addition to detecting subgingival calculus directly through the gingival tissue, SS-OCT can image calculus endoscopically (8). Kao et al. designed a miniature circumferential-scanning fiber-probe three-dimensional SS-OCT to distinguish between sound enamel (including enamel pearls) and dental calculus ex vivo. Utilizing imaging tools such as an OCT probe is essential to proper diagnosis in this case because naturally occurring formations such as enamel pearls are susceptible to misdiagnosis when using the conventional dental explorer or periodontal probe for calculus detection. In their study, Kao et al. imaged extracted human teeth through circumferential scanning using the fiber-probe, resulting in a $2 \mathrm{D}$ OCT image. A $3 \mathrm{D}$ OCT image was reconstructed using twenty non-overlapping $2 \mathrm{D}$ images. To quantifiably distinguish the calculus from sound enamel and enamel pearls, regions of interest (ROI) were drawn and respective standard deviations (SD) of the OCT signal intensities were observed. The population mean of SD found for calculus was statistically significantly larger compared to that of either sound enamel or enamel pearl $(\mathrm{P}<0.001)$. Meanwhile, the population mean of $\mathrm{SD}$ values for sound enamel and enamel pearl were not significantly different. Although the experiment was carried out ex vivo with extracted teeth, the fiber-probe was designed with a diameter of approximately $0.9 \mathrm{~mm}$ allowing for insertion into the gingival pocket (8). SS-OCT in dentistry has previously focused on early diagnoses of caries; however, the high resolution and deep penetration depths of this imaging technique reveal its promising use of noninvasively imaging subgingival calculus. The barriers to SS-OCT's transition into a clinical environment include the need to redesign the device to accommodate actual patients and its substantial increase in price compared to other imaging techniques.

\section{Fluorescence}

Fluorescence technology has been applied in dentistry since 

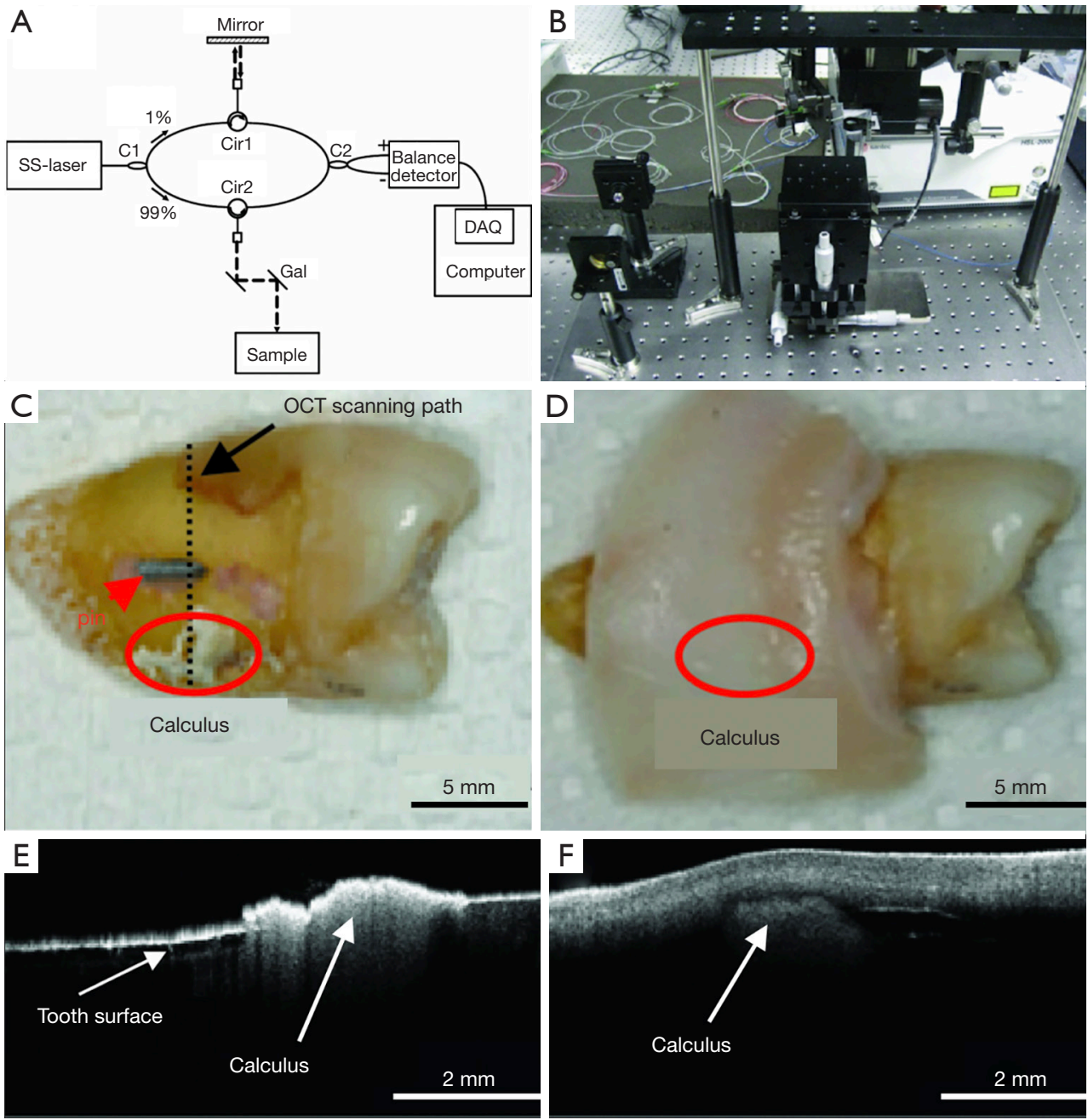

Figure 2 SS-OCT in vitro imaging of calculus. (A) Illustrated diagram of SS-OCT hardware components. (B) Image of SS-OCT and sample set-up. (C) Subgingival calculus sample without gingival tissue. (D) Gingival tissue, $0.8 \mathrm{~mm}$ thick, covering subgingival calculus. (E) SS-OCT image of subgingival calculus on the tooth root surface. (F) SS-OCT image of subgingival calculus underneath gingival tissue. Reprinted with permission under a Creative Commons (BY 4.0)—Gold open access license from (21). Copyright 2011 SPIE. DAQ, data acquisition; OCT, optical coherence tomography; SS-OCT, swept-source optical coherence tomography; SPIE, Society of Photo-Optical Instrumentation Engineers.

the early 1900s. It was first reported in dentistry in 1911 by Stubell who used ultraviolet light to observe the fluorescent properties of dentine and enamel in rabbits' teeth (24). Since then, the use of fluorescence technology in dentistry has expanded to restorations, carious lesion diagnosis, endodontics, and periodontics (24). Laser fluorescence spectroscopy and two-photon fluorescence microscopy has been used in periodontics to non-invasively detect subgingival calculus with high reproducibility (25).

The mechanism of fluorescence involves the excitation of a molecule in a low-energy state via absorption of incoming light. Once the molecule is in an excited state, it decays non-radiatively to vibrational states and eventually emits light to return to the low-energy ground state. Due to a phenomenon known as the Stokes shift, the emitted light has a longer wavelength than the exciting light (31). This was observed in Stubell's case in 1911 when rabbits' teeth emitted a blue light after being excited with UV light (24). The intensity and wavelength of the emitted light can describe the properties of the material. Previous investigations concluded that a combination of collagen and hydroxyapatite present in dental tissue was the main 
contributor to fluorescence (24). Furthermore, studies have revealed that bacteria-derived porphyrins were responsible for the fluorescent properties of supra- and subgingival calculus $(12,17,26,32)$.

A key aspect of using fluorescence to differentiate between subgingival calculus and adjacent soft and hard tissues is finding the optimal excitation wavelength. One study used $405 \mathrm{~nm}$ violet light via LEDs from a VistaCam ${ }^{\circledR}$ (Dürr Dental, Bietigheim-Bissingen, Germany) intra-oral camera system (17). In the study, groups of extracted teeth with dental caries, subgingival calculus, and supragingival calculus were analyzed; the effect of saliva and blood on the samples was also considered. In a clinical environment, the VistaCam ${ }^{\otimes}$ intra-oral camera would be inserted into the gingival sulcus to explore subgingival calculus deposits. The VistaCam ${ }^{\circledR}$ imaging system quantitatively determined the fluorescence intensity emitted from the samples via assigning fluorescence scores. Fluorescence scores, quantified by arbitrary fluorescence units, are established by the VistaCam system and influenced by variations in tooth samples and surface conditions. Greater fluorescence scores are associated with less fluorescence emitted from the sample. The study revealed greater fluorescence scores for supragingival (1.7-3.3) and subgingival (1.3-2.4) calculus compared to dental caries (0.9-2.2) and healthy teeth (0.9-1.1). Saliva and blood did not significantly affect the fluorescence scores. Although this device showed promising results, it was evaluated under in vitro conditions and was not designed for imaging within the gingival pocket. Therefore, alterations are needed to clinically image subgingival calculus (17).

Laser-based fluorescence with 635 or $655 \mathrm{~nm}$ excitation has been more commonly studied with regards to subgingival calculus detection. Kurihara et al. imaged extracted teeth with subgingival calculus and found that dentine caries emit a characteristic 700 and $720 \mathrm{~nm}$ emission when excited by 635 and $655 \mathrm{~nm}$ light, respectively. They further showed that $635 \mathrm{~nm}$ excitation is more capable of differentiating between subgingival calculus and dentin via differentiation ratios $\left(\mathrm{FI}_{\text {calculus }} / \mathrm{FI}_{\text {dentine }}\right.$, where FI is autofluorescence intensity) (27). The excitation wavelengths of 635 and $655 \mathrm{~nm}$ can be achieved by red laser diode systems such as AIGaInP (630-680 nm) and InGaAsP $(655 \mathrm{~nm})$ systems $(12,25,33)$. Kurihara et al. imaged subgingival calculus deposits on the root surfaces of extracted single- and multi-rooted teeth. By using a $633 \mathrm{~nm}$ He-Ne laser with a $700 \mathrm{~nm}$ high-path optical filter, they could visually differentiate between subgingival calculus and a sound root surface. Similar to the VistaCam ${ }^{\circledR}$ system, laser fluorescence would require insertion into the gingival sulcus in a clinical environment due to its inability to image through gingival tissue.

Krause et al. used a DIAGNOdent ${ }^{\mathrm{TM}}(\mathrm{KaVo}$, Biberach, Germany) system involving a $655 \mathrm{~nm}$ AIGaInP based red laser diode as the exciting light source to image subgingival calculus on extracted teeth in vitro. The following laserbased fluorescence mechanism is illustrated in Figure $3 A-\mathrm{a}$ laser is transmitted through a central quartz fiber and causes excitation on the root surface. The fluorescent light from the root surface is then collected by fibers concentric to the central fiber and transmitted to a photodiode detection unit. In comparison to the $\mathrm{VistaCam}^{\circledR}$ intra-oral camera system that had to be used in the dark, the DIAGNOdent ${ }^{\mathrm{TM}}$ system can operate under normal lighting situations (including reflected and ambient lighting) due to its long pass filter that only allows light greater than $680 \mathrm{~nm}$ to pass (25). The experimental sample included the extracted teeth that were partially covered by naturally occurring subgingival calculus and also covered in saline solution or human blood to mimic clinical situations. The DIAGNOdent ${ }^{\mathrm{TM}}$ system included a conventional probe (probe A) that must be altered to image within the gingival pocket (shown in Figure 3B). The proposed design of the altered periodontal probe includes a reflective surface inside the tip of the probe. This would allow the probe to be inserted parallel to the root surface, while the light source can reflect off of the reflective surface and be situated perpendicular and directly emitted to the root surface (25).

Krause et al. measured both the reproducibility and the ability to differentiate between the clean root surfaces and subgingival calculus. They found the laser-fluorescence values of the sound root surfaces and that of subgingival calculus to be $6.2 \pm 6.5$ and $57.7 \pm 34.1$ arbitrary units (AU), respectively. Figure $3 C$ reveals that one calculus sample actually had laser-fluorescence values around 100 , significantly distinguishing the calculus sample from the root surface. The laser-fluorescence values were measured after 15, 30 and $60 \mathrm{~min}$ and after 6 and $24 \mathrm{~h}$ to assure consistency over time. They then observed laserfluorescence values of the root surfaces before and after calculus removal and found significantly lower signals compared to the initial sample. These laser-fluorescence values were not affected by the saline solution and blood (25); however, other studies do not agree. For example, Kurihara et al. and Folwaczny et al. have both shown that blood and bacteria can weaken the emitted 

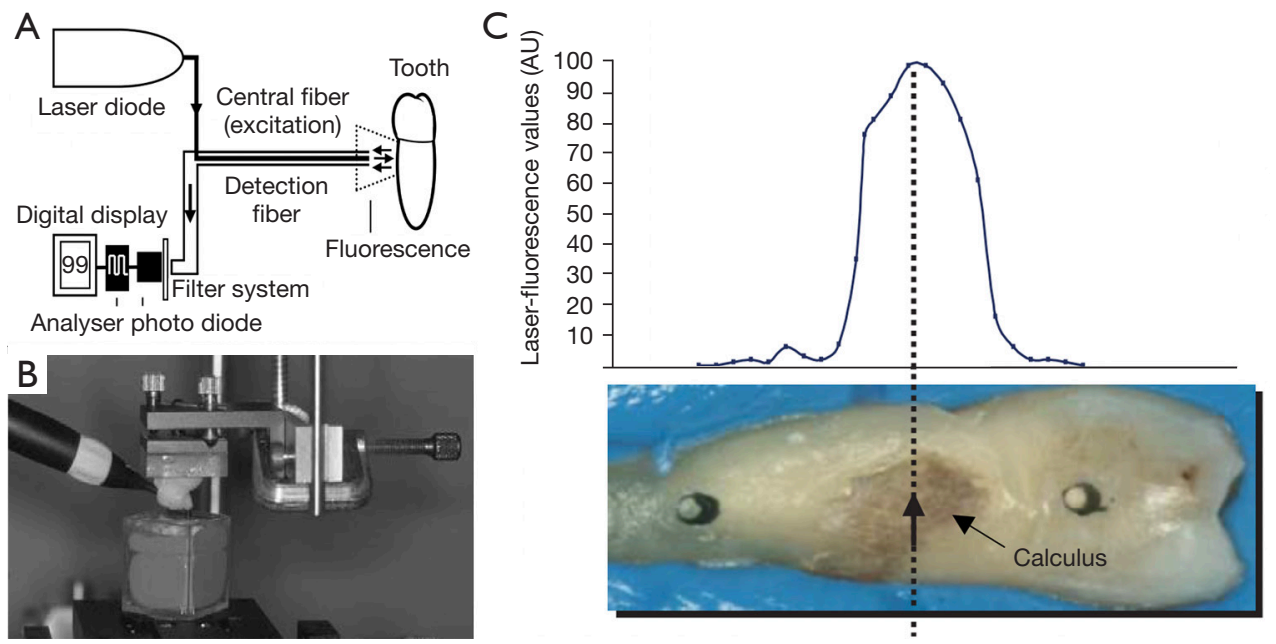

Figure 3 Laser fluorescence in vitro detection of subgingival calculus. (A) Diagram of the laser fluorescence system and components. (B) Image of laser fluorescence probe and sample. (C) Graph displaying laser-fluorescence values measured in AU versus the location of the tooth. Reprinted by permission from Springer, Lasers in Medical Science (25). AU, arbitrary units.

fluorescence of subgingival calculus because of increased photon scattering or absorbance $(12,27)$.

Although many studies confirm the use of laser fluorescence systems to detect subgingival calculus, few correlate the data to the actual surface area and volume of the subgingival calculus deposit on the root surface. Shakibaie et al. addressed this issue through their evaluation of three different $655 \mathrm{~nm}$ laser fluorescence systems (DIAGNOdent, DIAGNOdent Pen and KEY3) and the systems' abilities to detect subgingival calculus (33). These systems were used by two different operators with different skill levels on extracted posterior teeth ranging over seven different colors each with similar calculus colors. The apexes of the teeth were cemented into a typodont model with non-fluorescing silicone impression material in place of gingival tissue (33). After imaging, Shakibaie et al. then imaged the teeth using white light and ultraviolet reflectance. They used Image $J$ to determine the surface area and volume of the calculus deposits and compared that data to laser fluorescence readings from the three laser fluorescence systems. The readings more closely resembled the volume of the calculus deposit than the surface area because of the distribution of the fluorescing molecules throughout the calculus. However, laser fluorescence readings of calculus that are darker, or more opaque, would correlate more to the surface area than volume due to the inhibition of light penetration (33). The fluorescence values of all three systems positively correlated with the calculus volume; the KEY3's values were the most accurate. This may have been due to different internal signal amplification or due to the different optical tips: KEY3 used a chisel design, and DIAGNOdent and DIAGNOdent Pen both used a cylindrical tip. The limitations that stand out for these three systems and other laser fluorescence methods include variation in results due to operator skill, possible inaccuracy in results due to the use of a gingiva substitute, and longer times of operation compared to conventional subgingival calculus detection $(12,33)$.

Two-photon fluorescence microscopy offers several benefits to subgingival calculus imaging than one-photon fluorescence. The laser fluorescence methods mentioned above use an excitation wavelength within the visible spectrum and are one-photon fluorescence. In one-photon fluorescence, a single photon provides enough energy to produce fluorescence. However, these visible-range photons suffer from significant absorption and scattering by tissue. In contrast, two-photon techniques use near-infrared or infrared photons that suffer from less tissue absorption and scatter (15). In addition, this excitation only occurs at a single point where both photons overlap-therefore, it is possible to excite less background tissue for optical sectioning.

To image subgingival calculus using two-photon autofluorescence, Tung et al. used a time-correlated $800 \mathrm{~nm}$ Ti-sapphire laser and a $\times 20$ objective $(\mathrm{NA}=0.7)$. They compared this imaging method to one-photon fluorescence where they used a mercury lamp with a U-MNIBA2 filter cube to have an excitation wavelength 

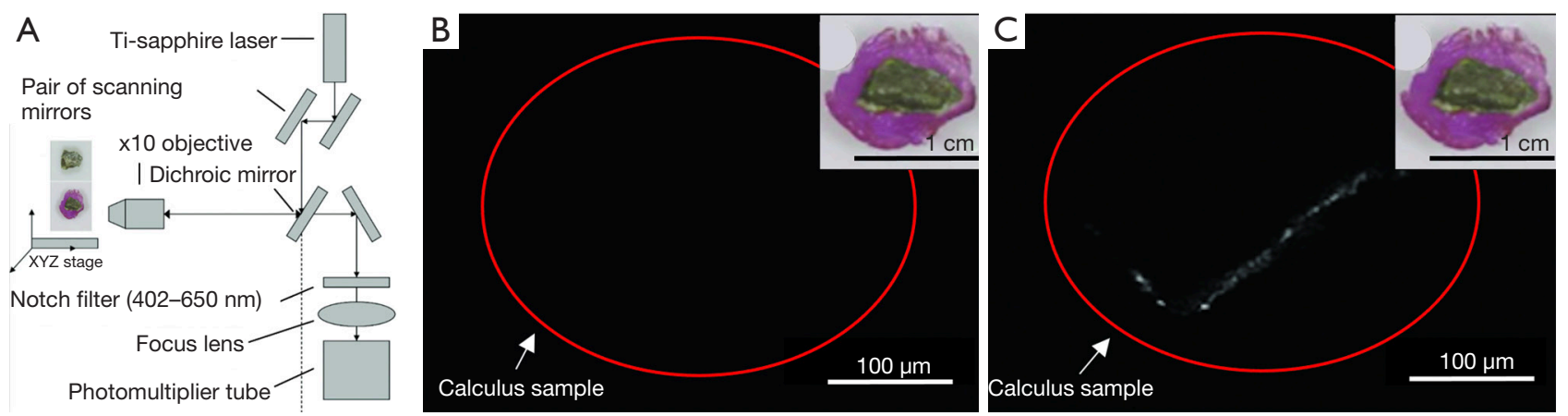

Figure 4 Two-photon autofluorescence in vitro imaging of subgingival calculus. (A) Diagram of the components of a two-photon autofluorescence system including Ti-sapphire laser, scanning mirrors, $\times 10$ objective, dichroic mirrors, notch filter (402-650 nm), focus lens, and a multiplier tube. (B) One-photon autofluorescence image of calculus sample with gingiva with picture of subgingival calculus sample covered with gingiva. (C) Two-photon autofluorescence image of calculus sample with gingiva with picture of subgingival calculus sample covered with gingiva. Reprinted with permission under a Creative Commons (BY 4.0)_Gold open access license from (3). Copyright 2011 SPIE. SPIE, Society of Photo-Optical Instrumentation Engineers.

between 470-490 $\mathrm{nm}$ and an emission wavelength between 510-550 nm (15). A key benefit of two-photon autofluorescence is the ability to image through gingival tissue. The samples were extracted teeth with identifiable subgingival calculus; a layer of gingival tissue was placed over the calculus to mimic clinical imaging. They reported that the improved penetration depth, optical sectioning, and limited scattering of two-photon autofluorescence resulted in fluorescence of only the subgingival calculus and very little scattering from the gingival tissue. There was no significant fluorescence from a healthy tooth using two-photon fluorescence. In contrast, the one-photon microscopy encountered image degradation due to out-offocus fluorescence (15).

Tung et al. followed up on their initial two-photon autofluorescence subgingival calculus imaging study using a $\times 10$ objective $(\mathrm{NA}=0.25)$ instead of the $\times 20$ objective. This allows for a longer working distance $(\mathrm{WD}=10.6 \mathrm{~mm})$ compared to the initial (WD $=1.30 \mathrm{~mm}$ ). A longer working distance of $10.6 \mathrm{~mm}$ allows for even better penetration depth. Similar to the first study (15), a time-correlated $790 \mathrm{~nm}$ Ti-sapphire with an average power of 10 to $20 \mathrm{~mW}$ was used as a light source for two-photon autofluorescence. The complete set-up of the two-photon system is shown in Figure $4 A$ and consisted of the Ti-sapphire laser, a pair of scanning and dichroic mirrors, a $\times 10$ objective, a notch filter $(402-650 \mathrm{~nm})$, focus lens, and a multiplier tube. For one-photon microscopy, a $488 \mathrm{~nm}$ argon laser was used.

The samples imaged in this study was extracted subgingival calculus covered by gingival tissue. The autofluorescence images from the study revealed that one-photon excitation causes a significant amount of fluorescence in gingiva and is also hindered by a shallow penetration depth (Figure 4B). Meanwhile, the two-photon autofluorescence images showed that the excitation beam can penetrate the $1 \mathrm{~mm}$ gingival tissue without causing fluorescence and has a calculus penetration depth of $800 \mu \mathrm{m}$ (Figure 4C) (3).

Tung et al. also examined the cellular response to the laser source by using the $790 \mathrm{~nm}$ femtosecond two-photon microscope of average power $13 \mathrm{~mW}$ on human gingival fibroblasts for less than 120 seconds in a single spot and concluded that the excitation beam was nondestructive (3). However, a study focusing on the cellular response of hamster ovary cells to near-infrared femtosecond laser pulses in two-photon microscopes revealed that complete cell destruction occurred at mean powers greater than $10 \mathrm{~mW}$ (34). The main difference between the two studies is that König et al. used embryonic cells, and Tung used mature and differentiated cells.

While laser fluorescence has been shown to successfully distinguish calculus deposits from clean tooth surfaces, it requires insertion into the gingival pocket and does not result in actual images. Although two-photon autofluorescence can be more expensive compared to laser fluorescence, two-photon autofluorescence has successfully demonstrated its ability to image subgingival calculus through a layer of gingival tissue with low scattering and 

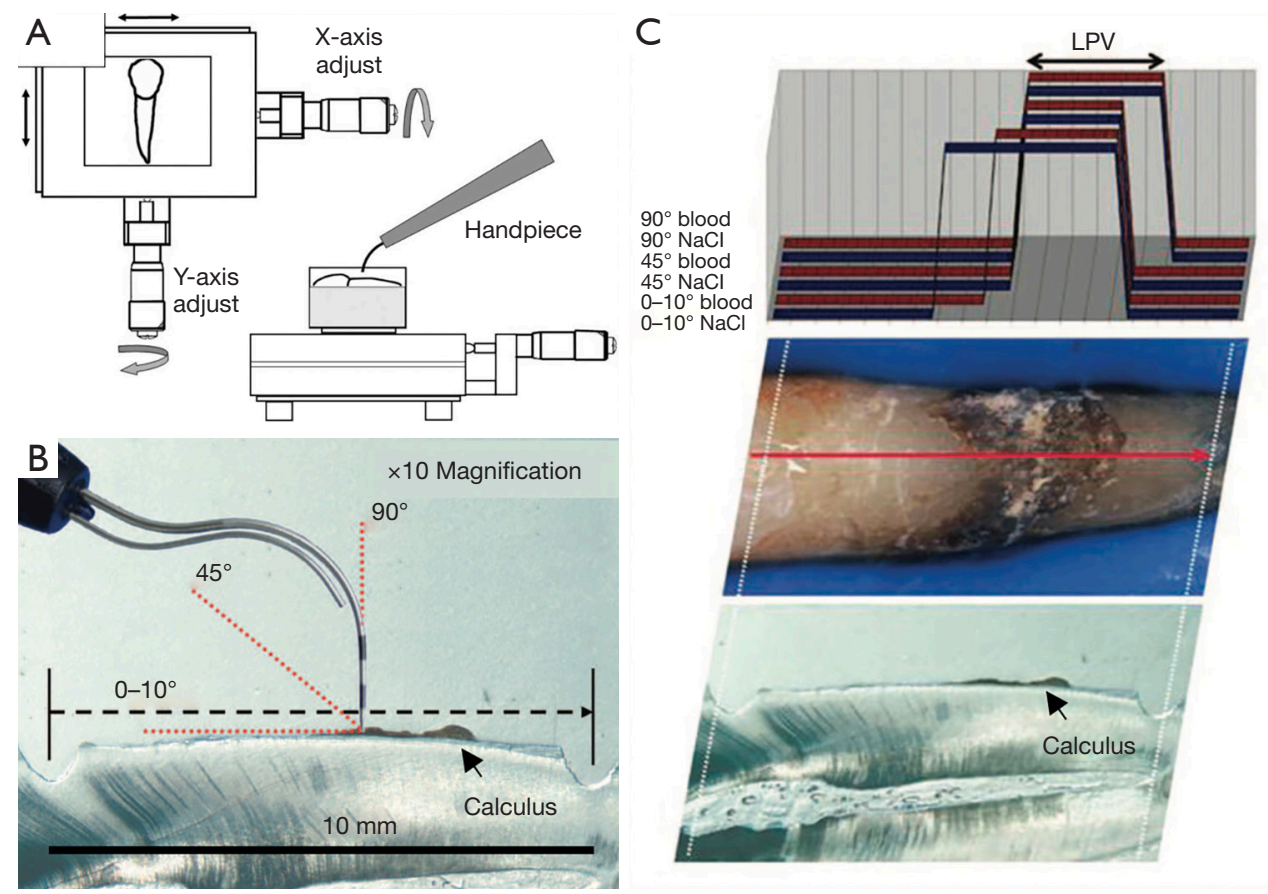

Figure 5 In vitro detection of subgingival calculus using an optical probe. (A) Diagram of the experimental setup including the adjustable platform and optical probe handpiece. (B) Image of the optical probe measuring calculus presence at angles of $0-10^{\circ}, 45^{\circ}$ and $90^{\circ}$. (C) The LPV measured by the DetecTar probe along the red line on the tooth and under various conditions of different angles and blood or $\mathrm{NaCl}$ solutions. Reprinted with permission from (10). Copyright 2005 John Wiley and Sons. LPV, length of successional positive values.

high optical sectioning.

\section{Differential reflectometry}

Differential reflectometry employs a similar mechanism to that of laser fluorescence to detect subgingival calculus. Differential reflectometry discriminates between two different surfaces by directing light onto the surfaces and then comparing the spectra of intensity of the respective reflected light (35). A system employing differential reflectometry, called DetecTar, is a $635 \mathrm{~nm}$ visible red LED optical probe that was designed to be inserted into the gingival sulcus to identify the spectral characteristics of subgingival calculus in terms of sensitivity and specificity of measurements (10). Greater sensitivity and specificity values refer to decreased numbers of false negatives and false positives, respectively. This study utilized the histological findings of calculus as the gold standard to which sensitivity and specificity were derived. This differential reflectometry system illuminates the root surface and uses an optical fiber to detect the reflected red LED radiation. The detected radiation is then transferred to a computer where an algorithm determines the presence of calculus $(10,28)$. The detection system also consists of an indicator component where a green light is flashed and a tone is played upon detection of calculus deposits (35). A general benefit of differential reflectometry compared to laser fluorescence and OCT is that LED-based optical probes that employ differential reflectometry is FDA-approved and already available for commercial use, whereas laser fluorescence and OCT systems for dental applications are still in experimental stages (10).

Krause $e t$ al. evaluated the differential reflectometry system's ability to detect subgingival calculus. The in vitro experiment as shown in the diagram in Figure $5 A$ included an adjustable platform, the DetecTar optical probe, extracted teeth with naturally occurring subgingival calculus, and a saline solution or human blood covering the root surface to simulate clinical situations. The DetecTar system carries out measurements by associating its detection of calculus with a positive value and an absence of calculus with a value of zero. The length of successional positive values (LPV) would, therefore, be the length of calculus across the surface of the tooth. In this study, these measurements taken by 
the DetecTar were compared to histological findings of calculus to determine the sensitivity and specificity of the measurements (10). To simulate clinical situations, Krause et al. measured the presence of calculus from angles of $0-10^{\circ}$, $45^{\circ}$ and $90^{\circ}$ (Figure 5B). The LPV measurements taken by the DetecTar also considered ambient blood or $\mathrm{NaCl}$ solutions (Figure 5C). It was determined that longer LPVs were recorded in the saline solution and at contact angles of $0^{\circ}$ to $10^{\circ}$ between the root surface and optical probe whereas shorter LPVs were observed in the blood solution at an angle closer to $90^{\circ}$. The LPVs were no indication of accuracy, as the purpose of LPVs is to observe the length of the calculus sample measured by the DetecTar system in comparison to histological findings. A major drawback of utilizing the binary LPV system is the inability to accurately quantify the amount of calculus present as the contact angle of the DetecTar probe or the sample's environment may alter the LPVs. The use of the optical probe at $45^{\circ}$ and $90^{\circ}$ provided the most accurate readings as those measurements revealed no statistical difference compared to the histological findings. In contrast, utilizing the probe at angles between $0^{\circ}$ and $10^{\circ}$ artificially elongate the LPVs. Sensitivity and specificity were both recorded as $100 \%$ regardless of blood or saline conditions when the optical probe was used perpendicular to the root surface (10). However, since the study showed that blood can hinder the imaging, the authors suggested that the gingival pocket be rinsed with saline solution before and during measurements (10).

In an in vitro comparison study between the application of differential reflectometry and conventional periodontal probing, differential reflectometry systems provided more accurate results (35). In this study, 30 extracted teeth with subgingival calculus were placed in a typodont with 10 to $15 \mathrm{~mm}$ of their roots exposed; a clear medium body polyvinyl siloxane impression material (Monet Clearbite2) was used to resemble gingival tissue (35). The mean values of sensitivity, specificity, and accuracy were significantly higher for the DetecTar system than for periodontal probing. Furthermore, the tooth type played a significant role in detection capabilities: both detection methods had higher specificity and accuracy on single-rooted teeth in comparison to multi-rooted teeth. As mentioned in Krause et al.'s study, the contact angle can significantly influence the performance of the DetecTar system. Multi-rooted teeth consist of furcations that have limited accessibility and demand smaller contact angles thus resulting in worse performance in both detection methods (10).

The differential reflectometry detection system, DetecTar, has also been studied in an in vivo clinical environment (28). Kasaj et al. evaluated 176 tooth surfaces in two separate groups: group A and group B. In group A, the LEDbased optical probe was used to detect subgingival calculus. In group B, the optical probe was used consistently alongside root surface debridement. In contrast to Krause et al., Kasaj et al. always performed the calculus detection at an angle of $10^{\circ}$ to $15^{\circ}$ due to the insertion of the probe into an intact gingival pocket. The data was presented on the basis of the presence or absence of calculus while comparing the optical probe measurements to that of a stereomicroscope at $\times 10$ magnification. In the comparison between the optical probe and the stereomicroscope, $9.1 \%$ of the cases were falsepositive, and $1.7 \%$ were false-negative. They concluded that these less-accurate results were due to the clinical setting and the lack of visual control compared to prior in vitro studies (28).

Differential reflectometry, or the use of an LED-optical probe, benefits from its commercial availability and highly sensitive and specific detection of calculus. However, like laser fluorescence, the optical probe must be inserted into the gingival pocket and can be negatively influenced by saliva and blood. Additionally, the device is most specific at an angle of $45^{\circ}$ or $90^{\circ}$, which would be highly difficult to achieve in the narrow gingival pocket.

\section{Emerging techniques: Raman, Fourier transform-infrared (FTIR), ultrasonography, photoacoustic, electrochemical, combination of techniques}

Additional methods such as Raman spectroscopy (36,37), FTIR spectroscopy (38), ultrasonography (39), photoacoustic ultrasound (PA-US) (40), electrochemical sensors $(41,42)$, and a combination of imaging techniques $(43,44)$ have the potential to detect subgingival calculus given further studies.

Raman spectroscopy is a nondestructive, highly specific and sensitive diagnostic method used to assess the composition of biological samples such as dental calculus (37). Specifically, Raman spectroscopy can provide details about the vibrational states of matter based on the inelastic scattering of light (45). In an early study, a microRaman spectrograph with an argon ion laser as a $515 \mathrm{~nm}$ excitation source was used to acquire Raman spectra of dental calculus. The spectra ranged from 330 to $1,150 \mathrm{~cm}^{-1}$ and had major peaks at 951 and $968 \mathrm{~cm}^{-1}$ indicating the presence of crystalline phosphate-based minerals in calculus (36). Although this study involves extracted calculus in an in vitro setting, it shows that Raman spectroscopy does 
have the potential to diagnose subgingival calculus due to its ability to identify characteristic molecular components within a biological sample. In fact, a portable fiber-optic Raman spectroscopic technique had previously been used to identify the components of cardiac calculus. It was found that calcium hydroxyapatite was a main constituent of both cardiac calculus and dental calculus (36,37). One limitation of Raman is its poor penetration through tissues. Thus, the imaging probe would have to be placed in the sulcus as opposed to on the gingival surface.

FTIR spectroscopy identifies the organic and inorganic constituents of a biological sample by measuring light absorption (46). A FTIR spectra of supragingival and subgingival calculus had been observed using a Nicolet 7199B spectrometer (38). Hou et al.'s study revealed that subgingival calculus can be distinguished from supragingival calculus using FTIR spectroscopy.

Tridimensional ultrasonography is another cost-effective, highly accurate, and non-invasive imaging technique that allows for the observation of subgingival calculus debridement over time (39). Chifor et al. demonstrated that a $40 \mathrm{MHz} 2 \mathrm{D}$ ultrasonographic image can be processed and volumetrically evaluated to visualize the cortical bone, gingival connective tissue, free gingival margin, root surface, tooth enamel, and gingival epithelium. In this way, Chifor et al. argues that a 3D method could outperform a 2D ultrasonographic imaging system by reducing operator dependency and enabling the visualization of an entire oral structure. The current limitations of ultrasonography include the need for an appropriately sized transducer, the distortions of images due to fillings and restorations, and for 2D ultrasonography, specifically, the difficulty of reproducing 2D ultrasonographic slices (39).

PA-US technology is capable of characterizing dental and periodontal structures and diseases (40). PA-US utilizes the photoacoustic effect, which is defined by acoustic vibrations released from irradiated material. The acoustic vibrations detected by transducers can then be used to reconstruct a $3 \mathrm{D}$ image based on the variation in absorption coefficients of the sample material. Moore et al. employed PA-US to observe pocket depths by using 680 and $800 \mathrm{~nm}$ excitation on a porcine dental sulcus irrigated with a food-grade contrast agent. The study revealed the capabilities of PA-US technology in imaging subgingival calculus as it was previously used to image through $2.00 \mathrm{~mm}$ pocket depths and a gingival thickness of $1.50 \mathrm{~mm}(40)$.

Instead of detecting subgingival calculus through direct imaging, electrochemical detection is a different approach that may be employed to image the dysbiosis and inflammation associated with the subgingival calculus (41). Dysbiosis, characterized by the overgrowth of Porphyromonas gingivalis ( $P$. gingivalis) bacteria in the saliva, is indicative of the presence of subgingival calculus (42). Evaluating the presence of $P$. gingivalis through saliva testing is non-invasive, operator independent, and can be used as a primary screening tool for periodontal disease in addition to an identification method for subgingival calculus. The limitation of this method is the inability to accurately detect the location of subgingival calculus within the gingival pocket. However, it enables the ability to monitor periodontal disease through $P$. gingivalis' association with average pocket depth and the amount of subgingival calculus present (41). Although these various detection methods have the potential to identify subgingival calculus, further studies and modifications are clearly needed to customize them for subgingival calculus detection.

Multiple imaging and detection methods have also been used in conjunction with one another to improve diagnostic assessments within the oral cavity. In previous studies, lightinduced autofluorescence (LIAF) (43) and quantitative light-induced fluorescence (QLF) (44) have been used supplementarily to OCT to diagnose caries. In addition to detecting plaque and calculus, LIAF specifically also allows for improved OCT probe alignment in the case that the subject moves during scanning (43). QLF improves upon OCT imaging by providing a more objective diagnoses of caries due to OCT assessment relying more on visual inspection (44). The combination of fluorescence and OCT imaging techniques has the potential to detect subgingival calculus more accurately than any of the techniques alone. However, such studies specific to subgingival calculus are required for further discussion as these past multimodal studies have mainly focused on the assessment of caries.

\section{Summary}

The technological advancement on subgingival calculus detection continues to improve in dental medicine. Foremost, improvements on the field visibility from apparatuses like periodontal endoscopy, SS-OCT, laser fluorescence, two-photon fluorescence, and LED optical probing already make some strong impacts. However, subgingival calculus, its surface characteristics, hinder the light source interactions (i.e., fluoresce absorbance, backscattering, light emission). Table 2 highlights the capabilities and limitations of each subgingival calculus 


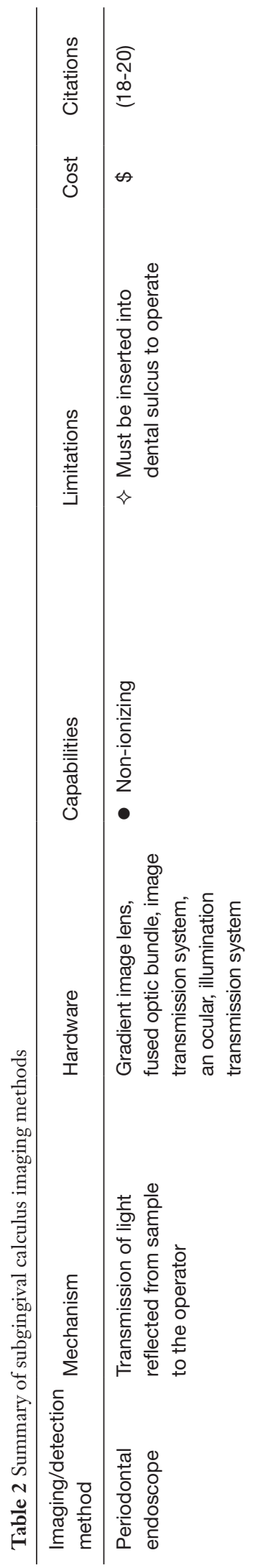

$\widehat{N}$
$\stackrel{N}{N}$
@
$\infty$
$\infty$

$\widehat{\bar{a}}$

$\stackrel{\leftrightarrow}{\leftrightarrow}$

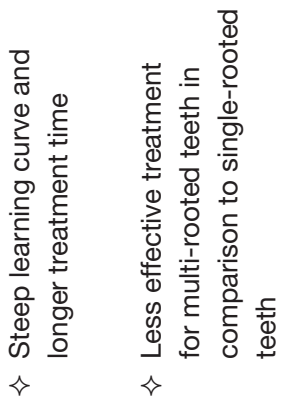

둥

$\frac{\pi}{\frac{\pi}{0}}$

व.

..ำ

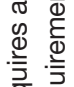

产
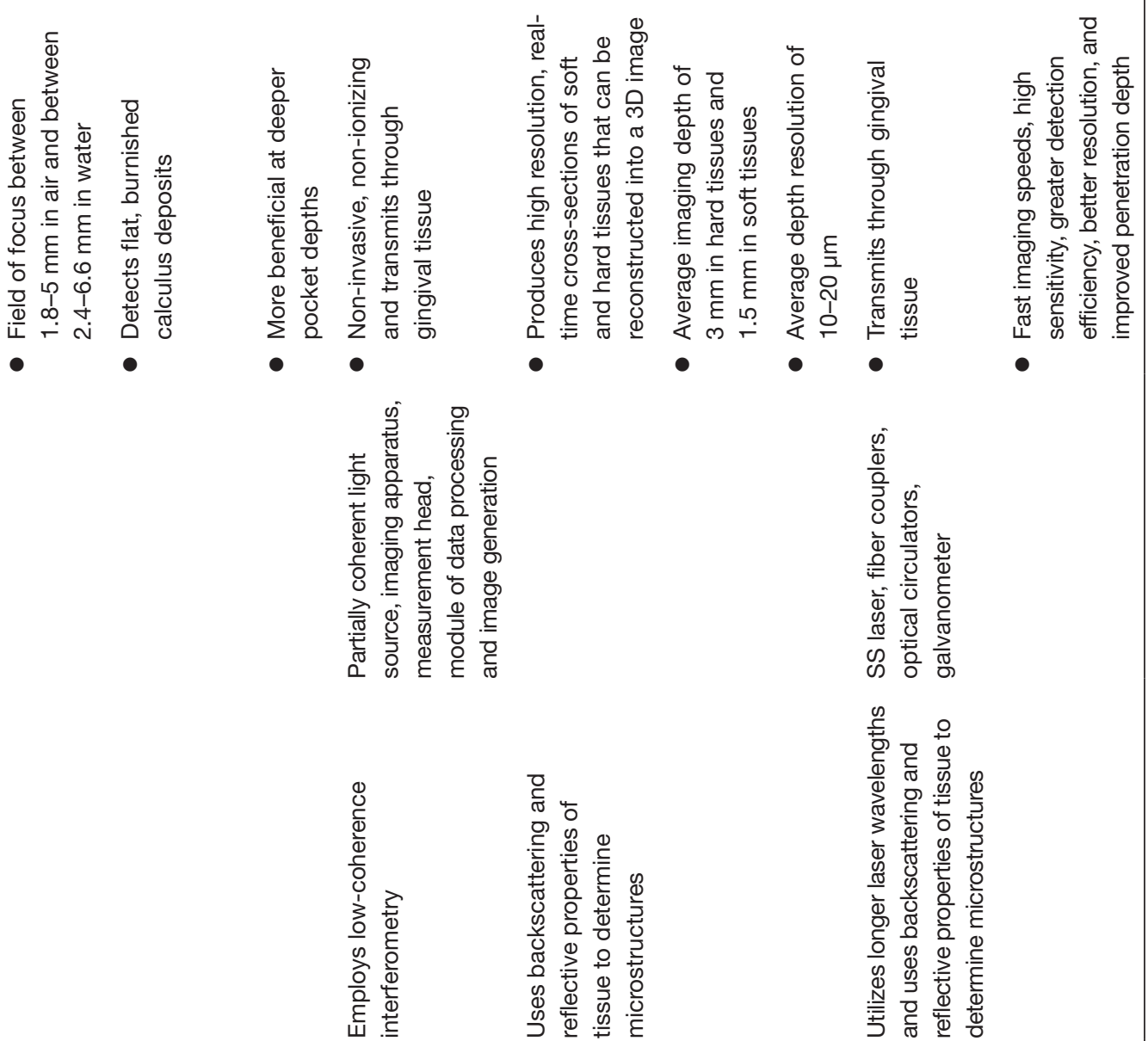

๖

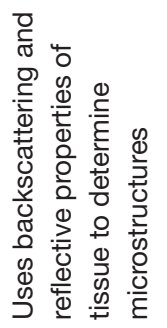

5
0
$i$
in
$\infty$ 


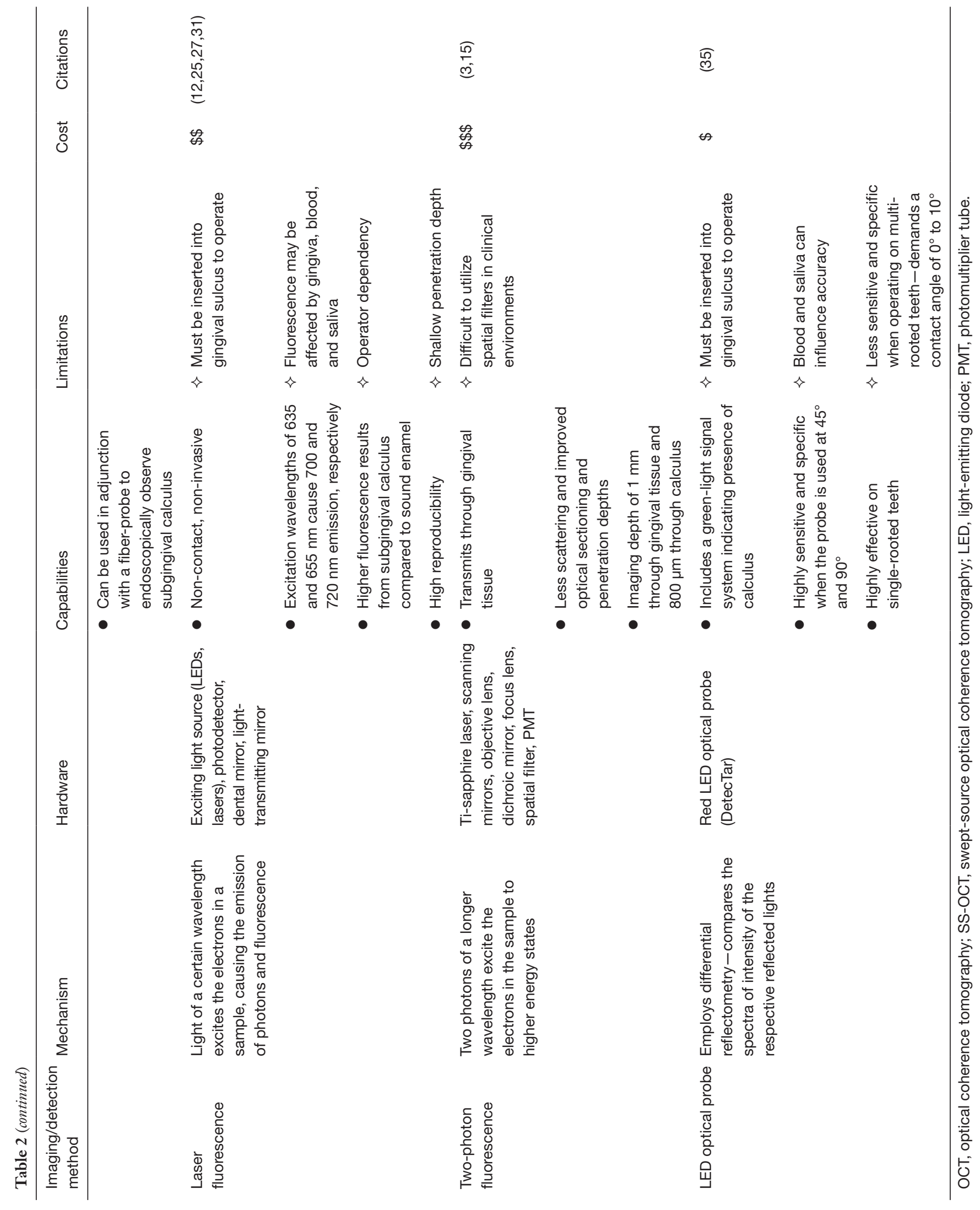


imaging and detection method. We may need to overcome the mechanical challenges presented from the subgingival calculus in order to reconstruct a diagnostic image.

Overall, an ideal detection method would be noninvasive, precise, accurate, cost and time effective, and have real-time visuals with a minimal learning curve. A further research may be needed to modify the detection systems to fit clinical environments.

\section{Acknowledgments}

Funding: The authors acknowledge NIH funding from National Institute of Dental and Craniofacial Research (R21 DE029917 to J.V.J., and R21 DE029025 to J.V.J.).

\section{Footnote}

Provenance and Peer Review: This article was commissioned by the Guest Editor (Mukund Seshadri) for the series "Advances in Oral and Maxillofacial Radiology" published in Frontiers of Oral and Maxillofacial Medicine. The article has undergone external peer review.

Reporting Checklist: The authors have completed the Narrative Review reporting checklist. Available at https://fomm. amegroups.com/article/view/10.21037/fomm-21-57/rc

Peer Review File: Available at https://fomm.amegroups.com/ article/view/10.21037/fomm-21-57/prf

Conflicts of Interest: All authors have completed the ICMJE uniform disclosure form (available at https://fomm. amegroups.com/article/view/10.21037/fomm-21-57/coif). The series "Advances in Oral and Maxillofacial Radiology" was commissioned by the editorial office without any funding or sponsorship. SK served as the unpaid editorial board member of Frontiers of Oral and Maxillofacial Medicine from October 2019 to September 2021. SK received presentation honoraria from Nobel Biocare and Neoss (unrelated to this manuscript) and he was a chair of the MIT Alumni board and the president of the ICP (no honorarium). JVJ reports receiving NIH funding from NIDCR under grants R21 DE029917 and R21 DE029025. JVJ is a founder of StyloSonics, LLC. The authors have no other conflicts of interest to declare.

Ethical Statement: The authors are accountable for all aspects of the work in ensuring that questions related to the accuracy or integrity of any part of the work are appropriately investigated and resolved.

Open Access Statement: This is an Open Access article distributed in accordance with the Creative Commons Attribution-NonCommercial-NoDerivs 4.0 International License (CC BY-NC-ND 4.0), which permits the noncommercial replication and distribution of the article with the strict proviso that no changes or edits are made and the original work is properly cited (including links to both the formal publication through the relevant DOI and the license). See: https://creativecommons.org/licenses/by-nc-nd/4.0/.

\section{References}

1. Lang NP, Lindhe J. editors. Clinical Periodontology and Implant Dentistry. 6th edition. Hoboken, NJ, USA: WILEY Blackwell, 2015.

2. Sherman PR, Hutchens LH Jr, Jewson LG, et al. The effectiveness of subgingival scaling and root planning. I. Clinical detection of residual calculus. J Periodontol 1990;61:3-8.

3. Tung OH, Lee SY, Lai YL, et al. Characteristics of subgingival calculus detection by multiphoton fluorescence microscopy. J Biomed Opt 2011;16:066017.

4. Tan BT, Mordan NJ, Embleton J, et al. Study of bacterial viability within human supragingival dental calculus. J Periodontol 2004;75:23-9.

5. Rabbani GM, Ash MM Jr, Caffesse RG. The effectiveness of subgingival scaling and root planing in calculus removal. J Periodontol 1981;52:119-23.

6. Anerud A, Löe H, Boysen H. The natural history and clinical course of calculus formation in man. J Clin Periodontol 1991;18:160-70.

7. Roberts-Harry EA, Clerehugh V. Subgingival calculus: where are we now? A comparative review. J Dent 2000;28:93-102.

8. Kao MC, Lin CL, Kung CY, et al. Miniature endoscopic optical coherence tomography for calculus detection. Appl Opt 2015;54:7419-23.

9. Mallya S, Lam E. White and Pharoah's Oral Radiology. 8th edition. Maryland Heights, MO, USA: Mosby, 2018.

10. Krause F, Braun A, Jepsen S, et al. Detection of subgingival calculus with a novel LED-based optical probe. J Periodontol 2005;76:1202-6.

11. Caffesse RG, Sweeney PL, Smith BA. Scaling and root planing with and without periodontal flap surgery. J Clin Periodontol 1986;13:205-10. 
12. Folwaczny M, Heym R, Mehl A, et al. The effectiveness of InGaAsP diode laser radiation to detect subgingival calculus as compared to an explorer. J Periodontol 2004;75:744-9.

13. Blue CM, Lenton P, Lunos S, et al. A pilot study comparing the outcome of scaling/root planing with and without Perioscope ${ }^{\mathrm{TM}}$ technology. J Dent Hyg 2013;87:152-7.

14. Prabhuji ML, Kishore HC, Chowdhary KY. Novel Systems For Calculus Detection And Removal-A Review. AOSR 2011;1:235-42.

15. Tung OH, Lee SY, Lai YL, et al. Detection of subgingival calculus through oral gum in vitro using two-photon fluorescence microscopy. Annu Int Conf IEEE Eng Med Biol Soc 2008;2008:4051-4.

16. Buchanan SA, Robertson PB. Calculus removal by scaling/root planing with and without surgical access. J Periodontol 1987;58:159-63.

17. Shakibaie F, Walsh LJ. Dental calculus detection using the VistaCam. Clin Exp Dent Res 2016;2:226-9.

18. Geisinger ML, Mealey BL, Schoolfield J, et al. The effectiveness of subgingival scaling and root planing: an evaluation of therapy with and without the use of the periodontal endoscope. J Periodontol 2007;78:22-8.

19. Stambaugh RV, Myers G, Ebling W, et al. Endoscopic visualization of the submarginal gingiva dental sulcus and tooth root surfaces. J Periodontol 2002;73:374-82.

20. Michaud RM, Schoolfield J, Mellonig JT, et al. The efficacy of subgingival calculus removal with endoscopyaided scaling and root planing: a study on multirooted teeth. J Periodontol 2007;78:2238-45.

21. Hsieh YS, Ho YC, Lee SY, et al. Subgingival calculus imaging based on swept-source optical coherence tomography. J Biomed Opt 2011;16:071409.

22. Colston B, Sathyam U, Dasilva L, et al. Dental OCT. Opt Express 1998;3:230-8.

23. Machoy M, Seeliger J, Szyszka-Sommerfeld L, et al. The Use of Optical Coherence Tomography in Dental Diagnostics: A State-of-the-Art Review. J Healthc Eng 2017;2017:7560645.

24. Mualla SK. Fluorescence And Dentistry. IOSR J Dent Med Sci e-ISSN 2016;15:65-75.

25. Krause F, Braun A, Frentzen M. The possibility of detecting subgingival calculus by laser-fluorescence in vitro. Lasers Med Sci 2003;18:32-5.

26. Buchalla W, Lennon AM, Attin T. Fluorescence spectroscopy of dental calculus. J Periodontal Res 2004;39:327-32.
27. Kurihara E, Koseki T, Gohara K, et al. Detection of subgingival calculus and dentine caries by laser fluorescence. J Periodontal Res 2004;39:59-65.

28. Kasaj A, Moschos I, Röhrig B, et al. The effectiveness of a novel optical probe in subgingival calculus detection. Int J Dent Hyg 2008;6:143-7.

29. Huang D, Swanson EA, Lin CP, et al. Optical coherence tomography. Science 1991;254:1178-81.

30. Yu H, Lee P, Lee K, et al. In vivo deep tissue imaging using wavefront shaping optical coherence tomography. J Biomed Opt 2016;21:101406.

31. Wolf DE. Fundamentals of fluorescence and fluorescence microscopy. Methods Cell Biol 2007;81:63-91.

32. Rams TE, Alwaqyan AY. In vitro performance of DIAGNOdent laser fluorescence device for dental calculus detection on human tooth root surfaces. Saudi Dent J 2017;29:171-8.

33. Shakibaie F, Walsh LJ. Surface area and volume determination of subgingival calculus using laser fluorescence. Lasers Med Sci 2014;29:519-24.

34. König K, So PT, Mantulin WW, et al. Cellular response to near-infrared femtosecond laser pulses in two-photon microscopes. Opt Lett 1997;22:135-6.

35. Shakibaie F, Walsh LJ. Differential reflectometry versus tactile sense detection of subgingival calculus in dentistry. J Biomed Opt 2012;17:106017.

36. Tsuda H, Arends J. Raman spectra of human dental calculus. J Dent Res 1993;72:1609-13.

37. Cheng CL, Chang HH, Lin SY. Spectroscopic study of chemical compositions of cardiac calculus using portable Raman analyzer with a fiber-optic probe. Biomed Spectrosc Imaging 2012;1:17-26.

38. Hou RZ, Wu JG, Soloway RD, et al. Fourier Transform Infrared Photoacoustic Spectroscopy of Dental Calculus. Mikrochim Acta 1988;95:133-6.

39. Chifor R, Badea AF, Chifor I, et al. Periodontal evaluation using a non-invasive imaging method (ultrasonography). Med Pharm Rep 2019;92:S20-32.

40. Moore C, Jokerst JV. Photoacoustic Ultrasound for Enhanced Contrast in Dental and Periodontal Imaging. In: Chan HL, Kripfgans OD. editors. Dental Ultrasound in Periodontology and Implantology. Cham: Springer Cham, 2021.

41. Yamanaka K, Sekine S, Uenoyama T, et al. Quantitative detection for porphyromonas gingivalis in tooth pocket and saliva by portable electrochemical DNA sensor linked with PCR. Electroanalysis 2014;26:2686-92.

42. Nichols FC, Rojanasomsith K. Porphyromonas gingivalis 
lipids and diseased dental tissues. Oral Microbiol Immunol 2006;21:84-92.

43. Le N, Subhash HM, Kilpatrick-Liverman L, et al. Noninvasive multimodal imaging by integrating optical coherence tomography with autofluorescence imaging for dental applications. J Biophotonics 2020;13:e202000026.

44. Maia AM, de Freitas AZ, de L Campello S, et al.

Evaluation of dental enamel caries assessment using Quantitative Light Induced Fluorescence and Optical

doi: $10.21037 /$ fomm-21-57

Cite this article as: Chang JJ, Chen C, Chang J, Koka S, Jokerst JV. A narrative review of imaging tools for imaging subgingival calculus. Front Oral Maxillofac Med 2023;5:4.
Coherence Tomography. J Biophotonics 2016;9:596-602.

45. Jayasooriya UA, Jenkins RD. Introduction to Raman Spectroscopy. In: Andrews DL, Demidov AA. editors. An Introduction to Laser Spectroscopy. Boston, MA, USA: Springer, 2002.

46. Hanke LD. Fourier Transform-Infrared Spectroscopy. Handb Anal Methods Mater 2014;19-20. Available online: https://www.mee-inc.com/hamm/fourier-transforminfrared-spectroscopy-ftir/ 